\title{
Prevalence and antibiotic resistance profile of enterobacteria involved in urinary infections in Bamako, Mali
}

\begin{abstract}
In Mali, urinary infection is one of the most common bacterial diseases in outpatients. Treatment of these diseases has become challenging due to the emergence of pathogens with increasing resistance to available antimicrobial agents. The aim of this study is to determine the prevalence and evaluate the antibiotic resistance profile of the most frequently isolated enterobacteria strains involved in urinary infections in Bamako, Mali. In this study, 1050 patients with clinical symptoms of urinary infection, referred to "Institut National de Recherche en Santé Publique (INRSP)" of Bamako, Mali, were evaluated between December 2010 and January 2011. The isolated bacteria were identified by biochemical tests. Disk diffusion method was applied to determine the antibiotic sensitivity of bacterial agents. Our results showed that 231(22\%) patients out of 1050 were shown to be urine culture positive $(70.6 \%$ females and $29.4 \%$ males). The most isolated entero-bacterium was E. coli with frequency rate of $62.3 \%$. The other enterobacteria were Klebsiella pneumoniae (19.5\%), Enterobacter cloacea (6.1\%), klebsiella oxycota (4.3\%) and Proteusmirabilis $(2.2 \%)$. All the enterobacteria isolated from urine specimens were sensitive to amikacin and colistin (92.9\%-100\%). The amikacin was more effective against all the uropathogen enterobacteria isolated in present study. According to the present survey, E. coli isolates were the predominant pathogens in urinary infections in the district of Bamako and were more prevalent in women than men. In the present study, the frequency of urinary pathogens was comparable to that reported in previous studies, showing an increasing resistance pattern to commonly prescribed antibiotics.
\end{abstract}

Keywords: enterobacteria, urinary infection, antibiotic resistance, ampicilin, tobramicin
Volume I Issue 4 - 2017

\author{
Mamadou Kanadjigui,' Amadou Hamadoun \\ Babana,' Brahima Traoré,' Fassé Samaké2 \\ 'Research Laboratory in Microbiology and Microbial \\ Biotechnology, University of Science, Techniques and Technology \\ of Bamako, Mali \\ ${ }^{2}$ Institute of Applied Sciences, University of Science, Techniques \\ and Technology of Bamako, Mali
}

\begin{abstract}
Correspondence: Amadou Hamadoun Babana, Research Laboratory in Microbiology and Microbial Biotechnology, University of Science, Techniques and Technology of Bamako, BP
\end{abstract} E 3206, Mali, Email ahbabana@laborem-biotech.com

Received: July 23, 2017| Published: August 03, 2017
Abbreviations: INRSP, institut national de recherche en santé publique; CFU, colony forming unit; $\mu \mathrm{g}$, microgram; CLSI, clinical and laboratory standards institute; E. coli, escherichia coli; K. pneumonia, klebsiella pneumonia; K. oxycota, klebsiella oxycota; E. cloacae, enterobacter cloacea; P. mirabilis, proteus mirabilis; AM, ampicilin; $\mathrm{AMO}+\mathrm{NA}$, amoxicillin+ nalidixic acid; $\mathrm{CH}$, chloremphenicol; $\mathrm{CF}$, cephalothin; CIP, ciprofleoxacin; NA, nalidixic acid; FM, furane; AN, amikacin; TOB, tobramicin; CRO, ceftriaxone; CTX, cefotaxime; CTZ, ceftazidine; GM, gentamicin; $\mathrm{CO}$, colistin

\section{Introduction}

The incidence of urinary tract infection in the world is about 150-250 million people worldwide Khosbakht et al. ${ }^{1}$ Tajbakhsh et al. ${ }^{2}$ Compared to men, urinary tract infections are more predominant among women as demonstrated by previous reports Wagenlehner et al. ${ }^{3}$ Mansour et al. ${ }^{4}$ According to many reports, E. coli accounts for approximately $75 \%$ of urinary tract infection reported although different microorganisms can cause urinary infection Ghorbani et al. ${ }^{5}$ Arabi et al. ${ }^{6}$ Administration of antibiotics is the mainly used to treat patients with symptomatic UTI. But; the excessive and improper use of antibiotics while feeding animals, indiscriminate prescription of antibiotics, and poor infection control strategies can in turn cause alterations in the gut commensal microbiota and eventually lead to the development of multidrug-resistant microorganisms Tajbakhsh et al. ${ }^{2}$ Considering the high recurrence rates and emergence of antibiotic resistance in uropathogens, knowledge about the area-specific prevalence of these pathogens and their antimicrobial resistance patterns is necessary. Also, in order to select more appropriate antibiotics and prevent therapeutic failures, it is important to determine uropathogens and their antibacterial susceptibility patterns. That is why the aims of this study were isolation of pathogenic agents involving UTI and determination of their antibiotics susceptibility pattern in patients referred to the "Institut National de Recherche en Santé Publique (INRSP)" of Bamako, Mali.

\section{Materials and methods}

\section{Sampling}

In total, 1050 patients with clinical symptoms of urinary infection referred to INRSP, Mali, were investigated. There were 537.6(51.2\%) females and 512.4(48.8\%) males, with an age range of 19-80years (mean, 36.8 years). Clean-Catch midstream urine of the patients was collected in a sterile tube $(4-5 \mathrm{ml})$ and immediately transported to the laboratory. For proper specimen collection, guidelines were given to all patients on a printed card Forbes et al. ${ }^{7}$

\section{Bacterial colony count of bacteria in urinary infections}

Using calibrated loop method, a measured amount of urine was inoculated to nutrient agar medium for colony count. After growth, equal or more than $10^{4} \mathrm{CFU} / \mathrm{ml}$ of potential pathogen(s) was interpreted as positive UTI and a result of $10^{2}-10^{4} \mathrm{CFU} / \mathrm{ml}$ was repeated. A less than $10^{2} \mathrm{CFU} / \mathrm{ml}$ was interpreted as negative UTI Mansour et al. ${ }^{4}$ Urine specimens were cultured for isolation of the bacterial agents of UTI on blood agar and MacConky agar media. All the bacteria isolated from urine in this study were identified using conventional biochemical tests MacFaddin et al. ${ }^{8}$ Mandell et al. ${ }^{9}$ 


\section{Antimicrobial susceptibility testing}

Antimicrobial susceptibility testing, in the present study, was done on Mueller-Hinton agar (Merck, Germany) using disk diffusion (Kirby Bauer's) technique. The procedure was performed according to Clinical and Laboratory Standards Institute (CLSI) guidelines to determine susceptibility of UTIs agents. ${ }^{10}$ The antibiotic disks comprised, ampicilin $(10 \mu \mathrm{g})$, kanamycin $(30 \mu \mathrm{g})$, cephalothin $(30 \mu \mathrm{g})$. Ciprofleoxacin $(5 \mu \mathrm{g})$, nalidixic acid $(30 \mu \mathrm{g})$, amikacin $(30 \mu \mathrm{g})$, tobramicin $(10 \mu \mathrm{g})$, ceftriaxone $(30 \mu \mathrm{g})$, cefotaxime $(10 \mu \mathrm{g})$ and gentamicin $(10 \mu \mathrm{g})$.

\section{Results and discussion}

In this study, 231(22\%) patients out of 1050 were showed to be urine culture positive (their colony count was equal or more than $10^{4}$ ). There were 163(70.6\%) females and 68(29.4\%) males in patients with urine positive culture. According to Foxman (2003), approximately 1 in 3 women will require antimicrobial treatment for urinary infections before age 24 , and $40 \%$ to $50 \%$ of women will have a urinary infection during their lifetime. In accordance with previous studies, prevalence of urinary infections was higher among females than males Tabasi et al. ${ }^{11}$ Tajbakhsh et al. ${ }^{2}$ This difference in the prevalence could be due to several factors, including anatomic differences and shorter path to the urethra and vagina, which causes pathogenic bacteria to have an easier passage to the bladder Tabasi et al. ${ }^{11}$ Ghanbari et al. ${ }^{12}$ The frequency of isolated bacteria and their relation to patient commune of residency and sex were given in (Figure 1A) (Figure 1B) respectively. In the present study, patients from the commune III of the district of Bamako was the less contaminated by the isolated pathogens, mainly E. coli and $K$. pneumonia (Figure 1B). The most common enterobacteria uropathogen was E. coli $(62.3 \%)$, followed by $K$. pneumoniae (19.5\%), E. cloacea (6.1\%), K. oxycota (4.3\%) and P.mirabilis $(2.2 \%)$ respectively. Similar observations have been reported in a previous study Fobes et al. ${ }^{7}$ Furthermore, in a study, E. coli was identified as the predominant cause of urinary infections $(58.28 \%)$, followed by $K$. pneumoniae $(11.65 \%)$ Ghanbari et al. ${ }^{12}$ Also, Ghanbari et al. ${ }^{12}$ demonstrated that E. coli was the most commonly isolated pathogen in both genders. In our study, the incidence of uropathogen infection was ranged in patients between 19-80(mean, 38.9) years old. The isolated bacterial pathogens showed wide differences in theirsusceptibility to the tested anti micro bialantibiotics. The relation between antimicrobial sensitivity patterns of the isolated bacteria in urine of the population studied is presented in (Table 1).

Based on results obtained from susceptibility testing (Table 1), all the bacteria recovered from urine specimens showed the highest degree of resistance to ampicillin and Amoxicillin+Nalidixic acid. The enteropatogens isolated from urine specimens were sensitive to amikacin and colistin (92.9\%-100\%). The amikacin was more effective against all the uropathogen enterobacteria isolated in present study. E. coli showed that this bacterium was sensitive to amikacin, furan $(97.9 \%)$, colistin $(99.3 \%)$, gentamicin, ceftriaxone, amikacin, $(90.5 \%)$ and to Ceftriaxone, Cefotaxime and Ceftazidine $(87.6 \%$ 93.1\%-91.7\%) respectively. Most of the isolated bacteria in this study were resistant to ampicillin (97.9\%) and Amoxicillin+Nalidixic acid cephalothin (51.49\%). In this study, we found that $P$. mirabilis strains isolated in urine specimens, contrary to other isolated enterobacteria, were resistant to furan and colistin $(60 \%)$. There are earlier studies in agreement to present finding Zhanel et al. ${ }^{13}$ Stratchounski et al. ${ }^{14}$ In present study a high level of bacterial resistance was seen to ampicillin. This is similar to previous studies in the United States Marchaim et al. ${ }^{15}$ The high prevalence of resistance to the commonly used antibiotics such as ampicillin, cephalothin and tetracycline has caused considerable alarm Olaitan, ${ }^{16}$ Orrett and Davis. ${ }^{17}$ The most effective antimicrobial agents in our study and other reported researches were amikacin and colistin Ghanbari ${ }^{12}$ for Gram negative bacilli. Based on the results of this study, it was revealed that the sensitivity of enterobacteria to ampicillin and other antibiotics was similar to many studies (Amin et al. ${ }^{18}$ Ghanbari et al. ${ }^{12}$ According to our results, the efficacy of amikacin was comparable to other reports Orrett and Davis ${ }^{17}$ Ghanbari et al. ${ }^{12}$

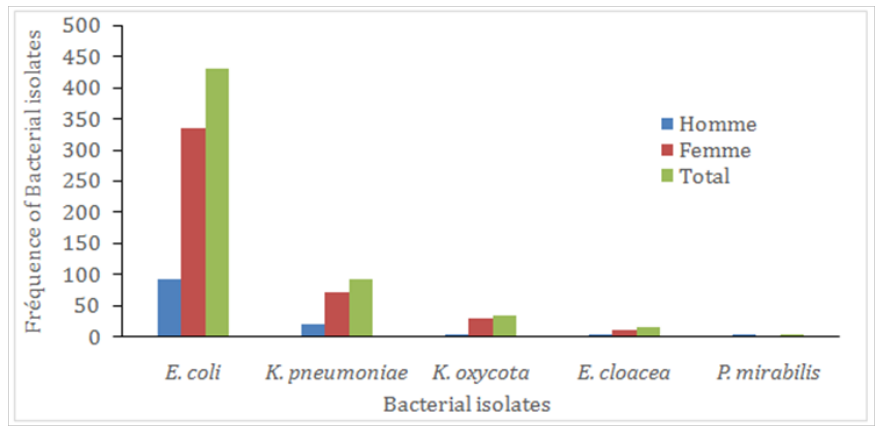

(A)

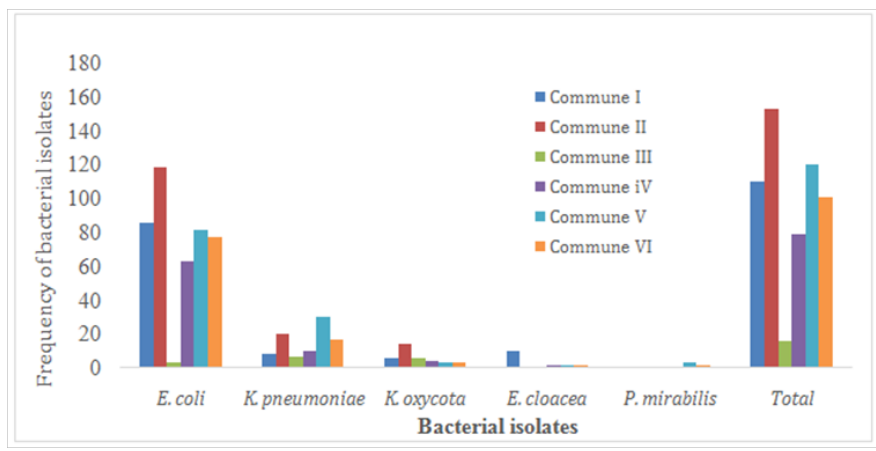

(B)

Figures I Frequency and type of pathogens isolated from urine specimens in males and females $(A)$ and the six communes of Bamako in Mali $(B)$.

Table I Antimicrobial resistance pattern profile of bacterial agents isolated from urine specimens of patients from Bamako district, Mali

\begin{tabular}{|c|c|c|c|c|c|c|c|c|c|c|c|c|c|c|}
\hline \multirow{2}{*}{ Isolates (number) } & \multicolumn{14}{|c|}{ Resistance rate (\%) } \\
\hline & AM & AMO+NA & CHL & CIP & CF & NA & CTZ & AN & FM & ТОВ & CRO & CTX & GM & $\mathrm{CO}$ \\
\hline E. coli $(n=430)$ & 91.7 & 67.7 & 34 & 28.5 & 17.4 & 31 & 8.3 & 2.1 & 2.1 & 24 & 12 & 6.9 & 13.2 & I \\
\hline K. pneumonia $(n=92)$ & 97.8 & 51.1 & 15.6 & 17.8 & 11.1 & 6.7 & 11.1 & 0 & 4.5 & 8.9 & 8.9 & 4.5 & 2.2 & 0 \\
\hline K. oxycota $(n=36)$ & 100 & 40 & 40 & 10 & 10 & 30 & 10 & 0 & 10 & 20 & 10 & 10 & 0 & 0 \\
\hline E. cloacea $(n=16)$ & 100 & 78.6 & 21.4 & 21.4 & 21.4 & 14.3 & 0 & 0 & 7.1 & 7.1 & 0 & 0 & 7.1 & 7 \\
\hline P. mirabilis $(n=5)$ & 100 & 60 & 40 & 0 & 0 & 20 & 0 & 0 & 60 & 0 & 0 & 0 & 0 & 60 \\
\hline
\end{tabular}

AM, ampicilin;AMO+NA, amoxicillin+ nalidixic acid; CHL, chloremphenicol; CF, cephalothin; CIP, ciprofleoxacin; NA, nalidixic acid; FM, furane;AN, amikacin; TOB, tobramicin; CRO, ceftriaxone; CTX, cefotaxime; CTZ, ceftazidine; GM, gentamicin; CO, colistin 


\section{Conclusion}

The most common isolated bacteria from urine specimens was $E$. coli and the most effective antimicrobial agents against the isolated enter bacteria were amikacin, Furan and colistin. It is concluded that Gram-negative bacilli (Enterobacteracea) were responsible for urinary infections and most of the strains were multi-drugs resistant.

\section{Acknowledgements}

None.

\section{Conflict of interest}

The author declares no conflict of interest.

\section{References}

1. Khoshbakht R, Salini A, Askhi HS, et al. Antibiotic susceptibility of bacterial strains isolated from urinary tract infections in Karaj, Iran. Jundishapur J Microbiol. 2012;6(1):86-90.

2. Tajbakhsh E, Tajbakhsh S, Khamesipour F. Isolation and molecular detection of gram negative bacteria causing urinary tract infection in patients referred to Shahrekord hospitals, Iran. Iran Red Crescent Med J. 2015;17(5):e24779.

3. Wagenlehner FM, Loibl E, Vogel H, et al. Incidence of nosocomial urinary tract infections on a surgical intensive care unit and implications for management. Int J Antimicrob Agents. 2006;28(S1):86-90.

4. Mansour A, Manijeh M, Zohreh P. Study of bacteria isolated from urinary tract infections and determination of their susceptibility to antibiotics. Jundishapur J Microbiol. 2009;3(4):118-123.

5. Ghorbani A, Ehsanpour A, Roshanzamir N, et al. Alterations in antibiotic susceptibility of urinary infection pathogens. J Nephropathol. 2012;1(1):43-48.

6. Arabi FMZ, Banazadehi A. Prevalence and antimicrobial susceptibility patterns of uropathogens among patients referring to valieasr laboratory in Najafabad, Isfahan. Iran Mid East J Sci Res. 2013;13(1):85-90.

7. Forbes BA, Sahm DF, Weissfeld AS. Bailey and Scott's Diagnostic microbiology. 12th ed. USA: Mosby Elsevier; 2007. p. 842-855.
8. MacFaddin JF. Biochemical tests for identification of medical bacteria. 3rd ed. Philadelphia, USA: Lippincott Williams and Wilkins; 2000.

9. Mandell GL, Bennett JE Dolin R. Principles and practice of infectious diseases. USA: Churchill Livingstone; 2005. p. 881-882.

10. Foxman B. Epidemiology of urinary tract infections: incidence, morbidity, and economic costs. Am J Med. 2002;113(S1A):5S-13S.

11. Tabasi M, AsadiKaram MR, Habibi M, et al. Phenotypic assays to determine virulence factors of uropathogenic escherichia coli (UPEC) isolates and their correlation with antibiotic resistance pattern. Osong Public Health Res Perspect. 2015;6(4):261-268.

12. Ghanbari F, Khademi F, Saberianpour S, et al. An epidemiological study on the prevalence and antibiotic resistance patterns of bacteria isolated from urinary tract infections in central Iran. Avicenna $J$ Clin Microb Infec. 2017;4(2):e46049.

13. Zhanel GG, Hisanaga TL, Laing NM, et al. Antibiotic resistance in outpatient urinary isolates: final results from the North American urinary tract infection collaborative alliance (NAUTICA). Int $J$ Antimicrob Agents. 2005;26(5):380-388.

14. Stratchounski LS, Rafalski VV. Antimicrobial susceptibility of pathogens isolated from adult patients with uncomplicated communityacquired urinary tract infections in the Russian Federation: two multi centre studies, UTIAP-1 and UTIAP-2. Int $J$ Antimicrob Agents. 2006;28(S1):S4-9.

15. Marchaim D, Navon-Venezia S, Schwaber MJ, et al. Isolation of imipenem-resistant Enterobacter species: emergence of KPC2 carbapenemase, molecular characterization, epidemiology, and outcomes. Antimicrob Agents Chemother. 2008;52(4):1413-1418.

16. Janet O Olaitan. Asymptomatic bacteriuria in female students population of a Nigerian university. Internet J Microbiol. 2006;2:2-6.

17. Orrett FA, Davis GK. A comparison of antimicrobial susceptibility profile of urinary pathogens for the years, 1999 and 2003. West Indian Med J. 2006;55(2):95-99.

18. Amin M, Mehdinejad M, Pourdangchi Z. Study of bacteria isolated from urinary tract infections and determination of their susceptibility to antibiotics. Jundishapur J Microbiol. 2009;2(3):118-123. 